\title{
The Clock Protein Bmal1 Regulates Circadian Expression and Activity of Sulfotransferase 1 a1 in Mice
}

\author{
Lianxia Guo, Fangjun Yu, Tianpeng Zhang, and Baojian Wu
}

Research Center for Biopharmaceutics and Pharmacokinetics, College of Pharmacy (L.G., F.Y., B.W.) and Guangdong Province Key Laboratory of Pharmacodynamic Constituents of TCM and New Drugs Research (L.G., T.Z., B.W.), Jinan University, Guangzhou, China

Received May 18, 2018; accepted July 23, 2018

\begin{abstract}
Sulfotransferase 1 1a1 (Sult1a1) is a phase Il enzyme that contributes extensively to metabolism and detoxification of various drugs and chemicals. Here we aimed to investigate a potential role of the clock protein Bmal1 (brain and muscle Arnt-like protein-1) in circadian regulation of Sult1a1 in mice. The regulatory effects of Bmal1 on Sult1a1 were assessed both in vivo (using Bmal1- deficient mice) and in vitro (using both normal and serum-shocked Hepa-1c1c7 cells). The relative mRNA and protein levels of Sult1a1 in the cells or mouse livers were measured by RT-qPCR and Western blotting, respectively. Sulfation activities of two Sult1a1 substrates (i.e., $p$-nitrophenol and galangin) were determined using mouse liver $\$ 9$ fractions. Transcriptional regulation of Sult1a1 by Bmal1 was investigated using luciferase reporter, electrophoretic mobility shift (EMSA), and chromatin immunoprecipitation (ChIP) assays. We first showed that hepatic Sult1a1 was rhythmically expressed at both
\end{abstract}

mRNA and protein levels (higher expressions during the night than the daytime). Consistently, the liver sulfation activities toward two Sult1a1 substrates were circadian time dependent with a higher activity at ZT14 than at ZT2. Furthermore, deletion of Bmal1 in mice blunted the circadian rhythmicity of hepatic Sult1a1 (with reduced expression levels). Likewise, Bmal1 positively regulated Sult1a1 expression in conventionally cultured Hepa-1c1c7 cells, and Bmal1 knockdown blunted expression rhythmicity of Sult1a1 in serum-shocked Hepa-1c1c7 cells. A combination of promoter analysis, EMSA and ChIP assays revealed that Bmal1 stimulated Sult1a1 transcription through its specific binding to the-571- to -554-bp region (an E-box element) in the promoter. In conclusion, Bmal1 activated the transcription of Sult1a1 and controlled circadian expression and activity of the enzyme.

\section{Introduction}

Sulfation (also known as sulfonation) is a phase II metabolic reaction that plays an important role in drug/chemical metabolism and detoxification (Gamage et al., 2006; James and Ambadapadi, 2013). The sulfation reaction turns the substrate molecules into hydrophilic products (i.e., sulfated metabolites or sulfates) that are generally biologically inactive and more excreteable (Chapman et al., 2004). The enzymes catalyzing the sulfation reaction are a superfamily of sulfotransferases (SULTs) that are divided into four families in humans, namely, SULT1 (eight members), SULT2 (three members), SULT4 (one member), and SULT6 (one member) (Blanchard et al., 2004). The main contributors to xenobiotic sulfation are usually from SULT1 and SULT2 families (Allali-Hassani et al., 2007). SULT1A1 is an important member of SULT1 family, with high expressions in the liver and intestine (Teubner et al., 2007; Riches et al., 2009). SULT1A1 contributes extensively to metabolism and detoxification of structurally and chemically diverse compounds, including drugs (e.g., acetaminophen, epinephrine, and minoxidil) and other chemicals

This work was supported by the National Natural Science Foundation of China [Grants 81722049 and 81573488 \}.

https://doi.org/10.1124/dmd.118.082503. [such as simple phenols (e.g., p-nitrophenol), polyphenols (e.g., flavonoids), and iodothyronines (e.g., 3,3',5-triiodothyronine)] (Glatt et al., 2001; Dong et al., 2012).

Mammalian physiology and behaviors are subjected to circadian rhythms (a rhythm of about 24 hours). Circadian rhythms are controlled by the circadian clock that is a hierarchical multi-oscillator system (Takahashi et al., 2008). The master clock (located in the hypothalamic suprachiasmatic nucleus) synchronizes the peripheral clocks (present in peripheral tissues) through neural and hormonal signals (Partch et al., 2014; Ono et al., 2015). At the molecular level, all circadian clocks consist of multiple core clock proteins [i.e., BMAL1 (brain and muscle Arnt-like protein-1), CLOCK (circadian locomotor output cycles kaput), PER (period), and CRY (cryptochrome)] that form a transcriptionaltranslational feedback loop system. BMAL1 heterodimerizes with CLOCK to activate the transcription of clock-controlled genes (CCGs), including PER and CRY (Reppert and Weaver, 2002). Once reaching critical levels, PER and CRY proteins inhibit the activity of BMAL1/ CLOCK dimer, thereby driving rhythmic expressions of CCGs (Akhtar et al., 2002). Additional factors such as REV-ERB proteins function to reinforce and maintain the robustness of circadian oscillators (Solt et al., 2011).

There is accumulating evidence that the expressions of drugprocessing genes \{including phase I [e.g., cytochrome P450 3A4

ABBREVIATIONS: Bmal1, brain and muscle Arnt-like protein-1; ChIP, chromatin immunoprecipitation assay; CLOCK, circadian locomotor output cycles kaput; CRY, cryptochrome; DBP, D-box binding protein; EMSA, electrophoretic mobility shift assay; FBS, fetal bovine serum; KO, knockout; PER, period; RT-qPCR, quantitative reverse transcription polymerase chain reaction; siBmal1, short interfering RNA for BMAL1; Sult, sulfotransferase; ZT, Zeitgeber time. 
(CYP3A4), CYP2E1, and CYP2D6], phase II (e.g., Sult1a1 and glucuronosyltransferase 2b34), and phase III genes (e.g., P-glycoprotein) $\}$ are subjected to circadian rhythms (Takiguchi et al., 2007; Murakami et al., 2008; Zhang et al., 2009; Lu et al., 2013; Košir et al., 2013). Circadian expressions of drug-processing genes are associated with time dependence of drug toxicity and tolerance (Sukumaran et al., 2010). For instance, hepatotoxicity of acetaminophen is more severe during the night than the daytime (Matsunaga et al., 2008; DeBruyne et al., 2014). Mitoxantrone toxicity closely depends on circadian dosing time, with the least toxicity at 16 hours after light onset (Lévi et al., 1994). Interestingly, circadian expressions of multiple phase I and III processing genes are attributed to the regulatory effects of one or more clock genes such as E4 promoter-binding protein 4, DBP (D-box binding protein), HLF (hepatic leukemia factor), and differentiated embryo chondrocyte-2 (Takiguchi et al., 2007; Murakami et al., 2008; Matsunaga et al., 2012). However, the molecular mechanisms for rhythmic expression of phase II enzymes such as SULT1A1 remain elusive.

As noted above, BMAL1 and CLOCK form the positive limb in generation of circadian gene expression (Partch et al., 2014). The BMAL1/CLOCK heterodimer essentially is a transcriptional activator that stimulates the transcription of target genes (such as PER and $C R Y$ ) via binding to the E-box elements present in promoter regions (Buhr and Takahashi, 2013). Although in most cases both BMAL1 and CLOCK are required in transactivation of clock-controlled genes, BMAL1 can independently regulate the expression of certain genes such as Dio2 (type II iodothyronine deiodinase) (Sawant et al., 2017). In addition to regulating circadian rhythms, BMAL1 has regulatory roles in the development of various diseases including hypertension, diabetes, dyslipidemia, and obesity (Shimba et al., 2011; Pappa et al., 2013; Richards et al., 2014).

Sult1al is one of three Sult genes showing diurnal expressions (Zhang et al., 2009). In this study, we aimed to investigate a potential role of Bmal1 in circadian regulation of Sult1a1 in mice. Sult1a1 expressions in the livers of wild-type and Bmal1-knockout mice were determined every 4 hours around the clock. Sulfation activities of Sultla1 substrates were assayed using liver S9 preparations. Regulatory effects of Bmal1 on Sutl1a1 were assessed using both normal and serum-shocked Hepa$1 \mathrm{c} 1 \mathrm{c} 7$ cells. The specific promoter region for Bmal1 binding was identified through a combination of luciferase reporter, mobility shift, and chromatin immunoprecipitation assays. We demonstrate for the first time that Bmal1 controls circadian expression of Sult1a1.

\section{Materials and Methods}

Materials. Anti-Sult1a1 antibody was purchased from OriGene Technologies (cat. no. TA501951; Rockville, MD). Antibodies against Bmal1 (cat. no. ab3350) and glyceraldehyde-3-phosphate dehydrogenase (cat. no. ab9485) were purchased from Abcam (Cambridge, MA). Anti-IgG antibody (cat. no. 7074) was purchased from Cell Signaling Technology (Danvers, MA). $p$-Nitrophenol was obtained from Shanghai Macklin Biotech (Shanghai, China). Galangin was obtained from Weikeqi Biotech (Sichuan, China). pRL-TK vector was purchased from Promega (Madison, WI). 3'-Phosphoadenosine-5'-phosphosulfate was purchased from Sigma-Aldrich (St. Louis, MO) ( $>95 \%$ purity). siRNA targeting mouse Bmall (named siBmall) was obtained from Transheep (Shanghai, China). $p$-Nitrophenol sulfate and galangin sulfate were synthesized according to the sulfation assay protocol and purified by chromatographic elution (Sun et al., 2015).

Animal Studies. All animal care and experimental procedures were in compliance with guidelines approved by the Institute of Laboratory Animal Science of Jinan University (Guangzhou, China). Bmall knockout (Bmal1-KO) mice on a C57BL/6J background were obtained from BangYao Biologic Technology Co., Ltd. (Shanghai, China). All mice, receiving food and water ad libitum, were housed in a temperature- and humidity-controlled room with a standard 12-hour light/dark cycle (light on at 7:00 AM and off at 7:00 PM). Wildtype ( 8 to 9 weeks of age, male, $n=6$ ) and Bmal1-KO mice ( 8 to 9 weeks of age, male, $n=5)$ were euthanized at each circadian time point (ZT2, ZT6, ZT10, ZT14, $\mathrm{ZT} 18$, and ZT22), and the livers were isolated, snap-frozen, and stored at $-80^{\circ} \mathrm{C}$ until processing for mRNA and protein analyses.

Plasmid Construction. Mouse Bmall cDNA (GenBank accession No NM_007489.4) were synthesized and cloned into the XhoI and BamHI sites of the expression vectors pcDNA3.1(-) (Biowit Technologies, Shenzhen, China). Various versions of Sult1a1 promoter luciferase constructs $(-2018 /+39,-660 /$ +39 , and $-400 /+39$ ) were synthesized and cloned into the Bglll and Hindlll sites of the blank pGL4.10 vector (Transheep). After verification by DNA sequencing, the plasmids were transformed into Esherichia coli JM109 and purified using EasyPure HiPure PlasmidMiniPrep kits (TransGen Biotech, Beijing, China).

Cell Culture and Transfection. Hepa-1c1c7 and HEK293 cells were purchased from the Cell Bank of the Chinese Academy of Sciences (Shanghai, China). The cells were grown in Dulbecco's modified Eagle's medium supplemented with $10 \%$ fetal bovine serum (FBS) at $37^{\circ} \mathrm{C}$ in a $5 \% \mathrm{CO}_{2}$ humidified atmosphere. After seeding for 24 hours, the cells were transfected with a 200-ng Bmal1 plasmid and/or 200-ng reporter plasmid using a JetPrime transfection kit (Polyplus Transfection, Ill kirch, France). In gene knockdown experiments, the cells were transfected with 100-nM siRNA using JetPrime (Polyplus Transfection). On the next day, the cells were collected for mRNA determination or luciferase activity measurements.

Serum Shock Experiments. Serum shock experiments were performed to induce circadian gene expression in cultured Hepa-1c1c7 cells as previously described (Matsunaga et al., 2012). In brief, Hepa-1c1c7 cells were grown in Dulbecco's modified Eagle's medium supplemented with 10\% FBS and transfected with siBmall (or short interfering RNA for negative control as the control) for 24 hours. This was followed by incubation in serum-free medium for 12 hours. On the day of serum shock, 50\% FBS was added for 2 hours, and then the cells were changed back to serum-free medium. The cells were harvested for RNA extraction at $0,4,8,12,16,20$, and 24 hours after the serum shock. The Sult1a1 mRNA levels were measured by RT-qPCR assays.

RT-qPCR Assay. Total RNA was extracted from mice livers with RNAiso Plus reagent (Takara Bio Inc., Shiga, Japan) and used as a template for reverse transcription. qPCR was performed using GoTaqR qPCR Master Mix (Vazyme, Jiangsu, China) as previously described (Zhao et al., 2018). Amplification reaction consisted of an initial denaturation at $95^{\circ} \mathrm{C}$ for 5 minutes, followed by 40 cycles of denaturation at $95^{\circ} \mathrm{C}$ for 15 seconds, annealing at $60^{\circ} \mathrm{C}$ for 30 seconds, and extension at $72^{\circ} \mathrm{C}$ for 30 seconds. Peptidyl-prolyl cis-trans isomerase B was used as an internal control. The relative changes in gene expression were derived using the $2^{-\Delta \Delta \mathrm{CT}}$ method. All primer sequences are summarized in Table 1.

Enzyme-Linked Immunosorbent Assay. Hepa-1c1c7 cells were seed into six-well plates and transfected with a $2-\mu \mathrm{g}$ Bmal1 plasmid (pcDNA3.1 as a control) or a 100-nM siBmall (short interfering RNA for negative control as a control) for 48 hours. Then, the culture media were removed and the cells were lysed in 150- $\mu$ l RIPA lysis buffer (Beyotime, Shanghai, China) supplemented with $1 \mathrm{mM}$ PMSF. After centrifugation, the supernatant was collected for Sult1a1 measurement using a mouse Sult1a1 enzyme-linked immunosorbent assay kit (MEIMIAN, Jiangsu, China) according to the manufacturer's instructions.

Luciferase Reporter Assay. HEK293 cells were seed into 48-well plates and transfected 200-ng of Sult1a1 luciferase reporter plasmid, 20-ng of pRL-TK vector (an internal control with renilla luciferase gene), and a fixed amount of expression plasmid (Bmal1 or pcDNA3.1). Twenty-four hours later, the medium was removed and the cells were lysed in $45 \mu$ l passive lysis buffer (Promega). The cell lysate was collected to determine the luciferase activities using the DualLuciferase Reporter Assay System and GloMax 20/20 luminometer (Promega). Firefly luciferase activities were normalized to renilla luciferase values and expressed as relative luciferase units.

Western Blotting. Western blotting was performed as previously described (Zhang et al., 2018). In brief, mouse liver homogenate or cell lysate (40 $\mu \mathrm{g}$ total protein) was loaded and separated on $10 \%$ SDS-polyacrylamide gels and transferred onto polyvinylidenefluoride membranes (Millipore, Bedford, MA). Protein bands were visualized by enhanced chemiluminescence, and the band density was analyzed by FlourChem software. Glyceraldehyde-3-phosphate dehydrogenase was used as a loading control. 
TABLE 1

Oligonucleotides used in this study

\begin{tabular}{|c|c|c|}
\hline Gene & Forward (5' to $\left.3^{\prime}\right)$ & Reverse (5' to $\left.3^{\prime}\right)$ \\
\hline \multicolumn{3}{|l|}{ Genotyping } \\
\hline Bmal1 & GCCCATGACCTCAGTGCCACA & CCGGCTTTAAGGCTATTGATTT \\
\hline \multicolumn{3}{|l|}{ RT-qPCR } \\
\hline Bmal1 & CTCCAGGAGGCAAGAAGATTC & ATAGTCCAGTGGAAGGAATG \\
\hline Dbp & ACATCTAGGGACACACCCAGTC & AAGTCTCATGGCCTGGAATG \\
\hline Ppib & TCCACACCCTTTTCCGGTCC & CAAAAGGAAGACGACGGAGC \\
\hline \multicolumn{3}{|l|}{ Plasmid construction } \\
\hline Sult1a1 $(-2018 /+39)$ & GCCTCGAGGATATCAAGATCTGCCAAAATGGATTCCTCAG & CAGTACCGGATTGCCAAGCTTGCCTCCCTCGGTGC \\
\hline Sult1a1 $(-660 /+39)$ & CCGGTACCTGAGCTCGCTAGCCAGCCTCTTGTCTTTG & CCGGTACCTGAGCTCGCTAGCCAGCCTCTTGTCTTTG \\
\hline Sult1a1 $(-400 /+39)$ & GCCTCGAGGATATCAAGATCTCCAGGCATCGCTG & CAGTACCGGATTGCCAAGCTTGCCTCCCTCGGT \\
\hline \multicolumn{3}{|c|}{ 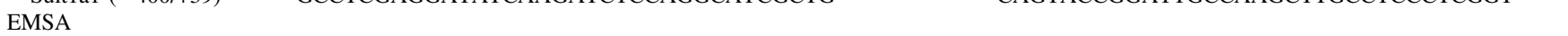 } \\
\hline Sult1a1 & TCTCCACCAGTCACGTGTGGTATTGGA & TCCAATACCACACGTGACTGGTGGAGA \\
\hline Sult1a1 (mutant) & TCTGCACCCGTATCACGTTGAATTGGA & TCCAATTCAACGTGATACGGGTGCAGA \\
\hline \multicolumn{3}{|r|}{ Fe } \\
\hline
\end{tabular}

Ppib, peptidyl-prolyl cis-trans isomerase B.

Electrophoretic Mobility Shift Assay. Hepa-1c1c7 cells were transfected with Bmal1 expression plasmid and then the nuclear proteins were extracted using the nuclear and cytoplasmic protein extraction reagents (Beyotime Biotech). Unlabeled and biotin-labeled probes were prepared by annealing complementary sense and antisense oligonucleotides (Table 1). EMSA assay was performed using the chemiluminescent EMSA kit (Beyotime Biotech). In brief, $6 \mu \mathrm{g}$ nuclear protein and $10 \mu \mathrm{M}$ unlabeled wide-type or mutated probes were mixed well in EMSA binding buffer and incubated at room temperature for 10 minutes. Then, a $0.2-\mu \mathrm{M}$ biotin-labeled probe was added to the reaction mixture and allowed another 20-minute incubation at room temperature. The DNA-protein complexes were loaded onto $4 \%$ nondenaturing polyacrylamide gels and subjected to electrophoresis, followed by transfer to the Hybond- $\mathrm{N}^{+}$membranes (Amersham, Buckinghamshire, UK). The products were visualized by using the enhanced chemiluminesence reagent and an Omega Lum G imaging system (Aplegen, Pleasanton, CA).

Chromatin Immunoprecipitation. ChIP assays were performed using a SimpleChIP Enzymatic Chromatin IP Kit (Cell Signaling Technology, Beverly, MA) as previously described (Lu et al., 2017). In brief, liver tissue was crosslinked with $37 \%$ formaldehyde, followed by quench with glycine and digestion with micrococcal nuclease. After sonication, chromatin fragments were incubated overnight with $10-\mu \mathrm{g}$ of anti-Bmall or normal rabbit IgG (negative control) at $4^{\circ} \mathrm{C}$. Protein $\mathrm{G}$ magnetic beads were then added to each ChIP sample and incubated for 2 hours. Beads were collected by placing the tubes in a magnetic separation rack and washed sequentially with low- and high-salt washes. Chromatins were eluted from the antibody/protein $\mathrm{G}$ magnetic beads with the elution buffer $\left(30\right.$ minutes, $\left.65^{\circ} \mathrm{C}\right)$. Crosslinking was reversed by incubation with $\mathrm{NaCl}$ and proteinase $\mathrm{K}$ for 2 hours at $65^{\circ} \mathrm{C}$. DNA was purified using spin columns and used as template for qPCR analyses using primers encompassing the Bmal1binding site (Table 1). The qPCR reaction consisted of an initial denaturation at $95^{\circ} \mathrm{C}$ for 5 minutes, followed by 50 cycles of denaturation at $95^{\circ} \mathrm{C}$ for 15 seconds, annealing at $60^{\circ} \mathrm{C}$ for 30 seconds, and extension at $72^{\circ} \mathrm{C}$ for 30 seconds.

Sulfation Assay. The livers were collected from wild-type and Bmall-KO mice euthanized at ZT2 and ZT14. Liver S9 fraction were prepared by centrifugation at $9000 \mathrm{~g}$ for 10 minutes (Richardson et al., 2016). Liver sulfation activity was determined using an incubation method as previously described (Wang et al., 2017). In brief, liver S9 fraction (0.6 mg total protein), $3^{\prime}$-phosphoadenosine-5' -phosphosulfate $(50 \mu \mathrm{M})$, and $p$-nitrophenol $(2 \mu \mathrm{M})$ or galangin $(4 \mu \mathrm{M})$ were incubated in potassium phosphate buffer $(50 \mathrm{mM}, \mathrm{pH} 7.4)$ at $37^{\circ} \mathrm{C}$ for 3 hours. The total volume of the incubation mixture was $200 \mu$ l. The reaction was terminated by the addition of $100 \mu \mathrm{l}$ ice-cold methanol (containing internal standard), followed by vortex and centrifugation (15 minutes, 13,000 g). The supernatant was collected and subjected to ultra-performance liquid chromatography/quadrupole time-of-flight mass spectrometry analysis.
Quantification of Sulfates. Sulfated metabolites were quantified using an ACQUITY ultra-performance liquid chromatography/quadrupole time-of-flight mass spectrometry system (Waters, Milford, MA) and an ACQUITY BEH C18 column $(2.1 \times 50 \mathrm{~mm}, 2.6 \mu \mathrm{m}$; Waters $)$. The mobile phase was $0.1 \%$ formic acid (mobile phase A) and $0.1 \%$ formic acid in acetonitrile (mobile phase B). The gradient elution program was $10 \% \mathrm{~B}$ at 0 to 1 minute, $10 \%-90 \% \mathrm{~B}$ at $1-3.5$ minutes, $90 \%$ B at 3.5-4.5 minutes, and $90 \%-10 \%$ B at $4.5-5$ minutes. The flow rate was set at $0.3 \mathrm{ml} / \mathrm{min}$. Mass spectrometer was operated at the negative ion full scan mode. Peak areas of $p$-nitrophenol sulfate and galangin sulfate were recorded with the exact mass of $\mathrm{m} / \mathrm{z} 218.8 \pm 0.05$ and $348.9 \pm 0.05 \mathrm{Da}$, respectively.

Statistical Analysis. Data are presented as mean \pm S.D. A two-way analysis of variance was performed to determine significant differences between groups (time $\times$ siBmall treatment). One-way analysis of variance was used to analyze gene/protein differences across several circadian time points. All other analyses were performed using the Student's $t$ test. The level of significance was set at $P<0.05$.

\section{Results}

Circadian Expression and Activity of Sult1a1 in Mouse Liver. The Sult1a1 mRNA in mouse liver displayed a significant circadian fluctuation (Fig. 1A). In general, the mRNA levels were higher during the night than the daytime (Fig. 1A). Also, hepatic Sult1a1 protein showed a circadian rhythm with a zenith at ZT18 (Fig. 1B). In line with circadian mRNA pattern, Sultla1 protein levels during the night were higher than those in the daytime (Fig. 1B). The sulfation activity of hepatic Sult1a1 was determined using $p$-nitrophenol and galangin as the substrates (Frame et al., 2000; Otake et al., 2002). Consistent with the protein levels, hepatic Sult1a1 showed a higher sulfation activity toward both $p$-nitrophenol and galangin at ZT14 than at ZT2 (Fig. 1C).

Bmal1 Deletion Blunts Circadian Rhythmicity of Hepatic Sult1a1. We first showed that Bmal1 was absent in Bmal1 knockout (Bmal1-KO or KO) mice by PCR analyses of the tails from the genetic mice (Fig. 2A, primers provided in Table 1). RT-qPCR analyses further confirmed that Bmal1-KO mice did not express Bmall mRNA in the liver and that Dbp (a target gene of Bmal1, Stratmann et al., 2012) was markedly downregulated (Fig. 2B). Genetic deletion of Bmall downregulated Sultla1 mRNA levels in the liver and blunted its circadian rhythmicity (Fig. 2C). Consistent with the mRNA changes, hepatic Sult1a1 protein was significantly reduced, and its rhythmicity was 
A

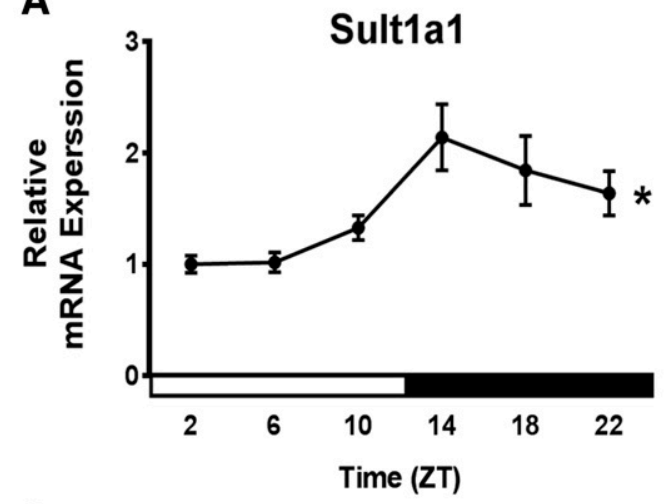

C

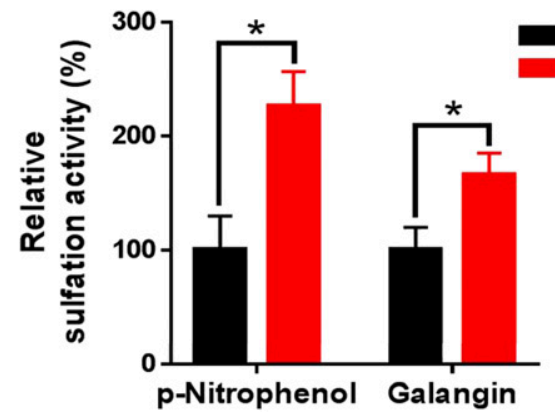

$\begin{array}{llllllll}\text { B } & \text { ZT } & 2 & 6 & 10 & 14 & 18 & 22\end{array}$
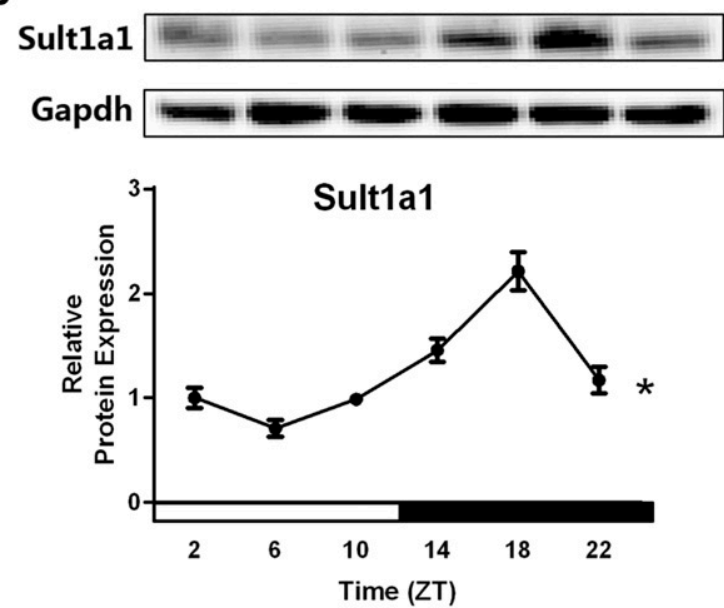

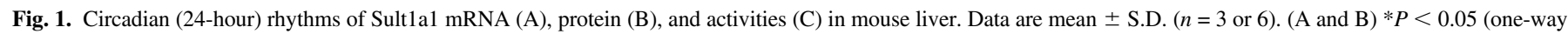

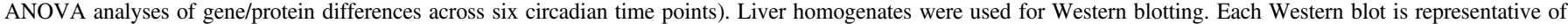
three independent experiments. ${ }^{*} P<0.05$ ( $t$ test). Unshaded box represents the light period and the shaded box represents the dark (lights off) period.

markedly dampened in Bmal1-KO mice (Fig. 2D). Furthermore, hepatic Sult1a1 activity at ZT14 was downregulated consistent with the enzyme expression changes (Fig. 2E). It was also noted that the sulfation activity differences ceased to exist due to Bmal1 deletion (Fig. 2E). These data strongly indicated that Bmal1 regulated circadian expression of Sult1a1.

Bmal1 Knockdown Blunts Sult1a1 Rhythmicity in SerumShocked Hepa-1C1c7 Cells. We next investigated the regulatory effects of Bmal1 on Sult1a1 using mouse hepatoma Hepa-1c1c7 cells. Overexpression of Bmal1 led to upregulation of Sult1a1 mRNA and protein (Fig. 3A). Consistent with this activation effect, siRNA targeting Bmal1 (named siBmal1) caused reductions in Sult1al mRNA and protein (Fig. 3B). It was noteworthy that Sultla1 protein levels in cell samples were determined by enzyme-linked immunosorbent assay rather than Western blotting. The exact reasons as to why Western blotting failed to detect cellular Sult1a1 were unknown. Low expression of Sult1a1 in the cells may be a contributing factor. A serum shock did generate rhythmic expressions of both Bmal1 and Sult1a1 (Fig. 4). As expected, siBmal1 effectively decreased mRNA levels of Bmal1 during a 24-hour period after serum shock (Fig. 4A). Interestingly, Bmal1 knockdown blunted rhythmic expression of Sult1a1 in Hepa-1c1c7 cells (Fig. 4B), confirming a critical role of Bmal1 in circadian regulation of Sult1a1.

Bmal1 is a Transcriptional Activator of Sult1a1. A Sult1a1 luciferase reporter (with $-2.0 \mathrm{~kb}$ proximal promoter) was first constructed to investigate a potential role of Bmall in transcriptional regulation of Sult1a1. The Bmall expression plasmid dose-dependently enhanced the promoter activity of Sult1a1, suggesting a positive role of Bmal1 in transcriptional regulation of Sult1a1 (Fig. 5A). Consistently, siBmal1 was found to significantly reduce the transcriptional activity of Sult1a1 (Fig. $5 B)$. Sequence analysis of mouse sult1a1 promoter revealed three potential Bmal1 binding sites (i.e., E-box elements, located at $-800 /-780,-571$ / -554 , and $-343 /-320$ bp) (Fig. 5C). Accordingly, two shorter (truncated) promoter constructs with the deletion of one or two E-box elements (i.e., $-0.66-$ and $-0.4-\mathrm{kb}$ constructs) were prepared for pinpointing the exact site for Bmal1 binding (Fig. 5C). Bmall increased the promoter activities of both 2.0- and 0.66-kb constructs (Fig. 5C). However, the activity of $0.4-\mathrm{kb}$ construct was unaffected in the presence of Bmall (Fig. $5 \mathrm{C})$. Furthermore, mutation of the E-box at $-571 /-554$ bp led to loss of luciferase reporter activity (Fig. 5C). These data suggested that the Bmal1 binding site was located between -0.66 and $-0.4 \mathrm{~kb}$ (probably the -571 / -554-bp region).

Next, electrophoretic mobility shift assay (EMSA) was performed to determine the binding potential of Bmal1 protein to Sult1a1 promoter using a biotin-labeled oligonucleotide (probe) encompassing the -571/-554-bp region. The biotin-labeled Sult1a1 probe was able to form a DNAprotein complex with Bmal1 (Fig. 6A). The complex band disappeared in the presence of unlabeled probe, but reappeared in the presence of mutated unlabeled probe (Fig. 6A). The results indicated that Bmal1 can bind directly to the $-571 /-554-$ bp region of Sult1al promoter.

Chromatin immunoprecipitation assay (ChIP) was further performed to determine potential in vivo interactions of Sult1a1 promoter with hepatic Bmal1 protein. The sequence encompassing the $-571 /-554-b p$ region (an E-box element) was amplified from the precipitated chromatin DNA (Fig. 6B). The Sultla1-E-box enrichment for antiBmal1 sample was markedly higher than that for anti-IgG sample, indicating significant recruitment of Bmal1 protein to Sult1a1-E-box (Fig. 6B). Similar results were observed for Dbp (a target gene of Bmal1) (Stratmann et al., 2012) (Fig. 6B). Taken together, our data indicated that Bmal1 transactivated Sult1a1 through its specific binding to the -571 to -554-bp region (an E-box element) in the promoter region.

\section{Discussion}

In this study, we showed that the expression and activity of hepatic Sult1a1 enzyme were circadian time dependent (Fig. 1). More importantly, we for the first time demonstrated that the clock protein 
A Marker $\mathrm{KO}+/-$ WT

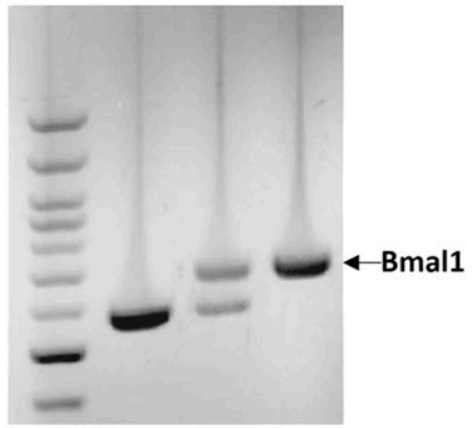

D

WT

\begin{tabular}{llllll}
\hline ZT & 6 & 10 & 14 & 18 & 22
\end{tabular}

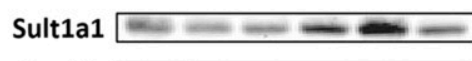

Gapdh

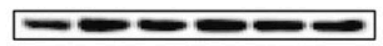

B
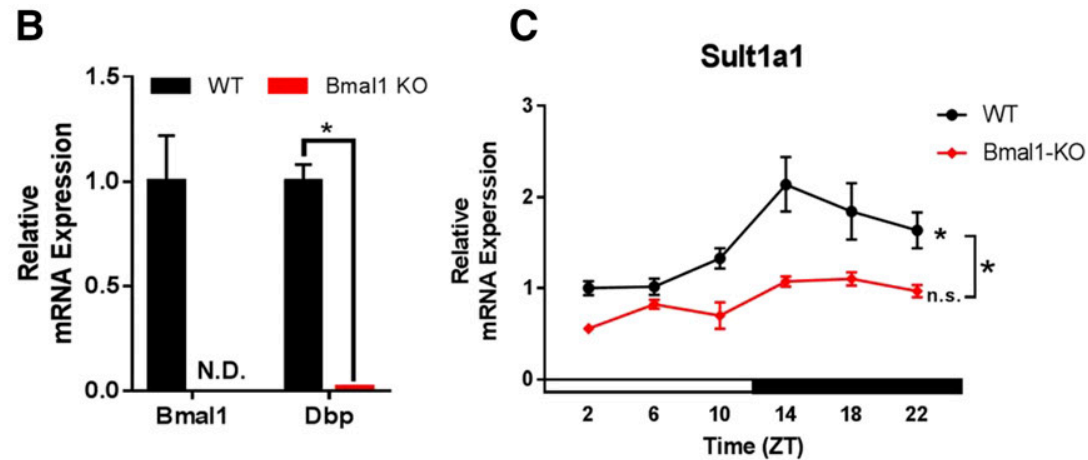

E
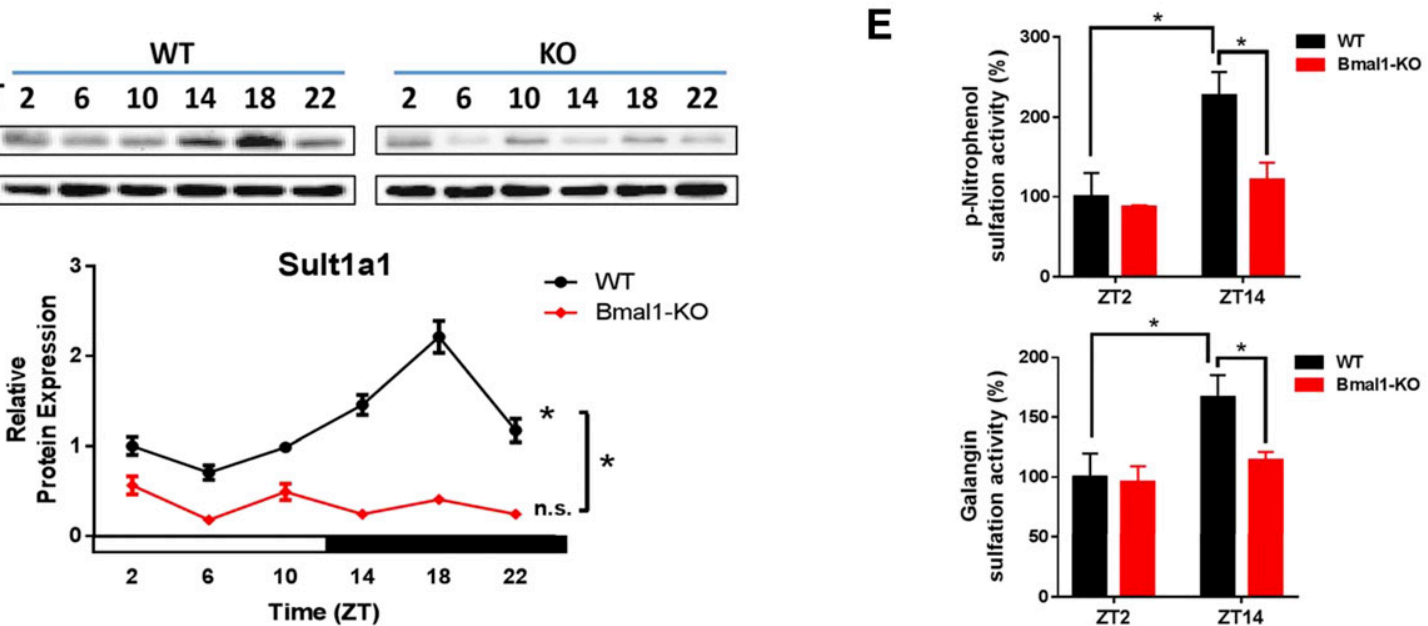

A
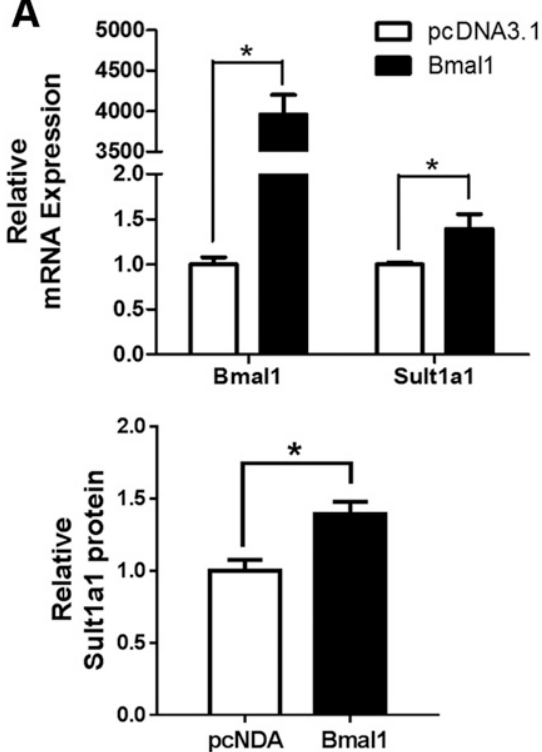

B
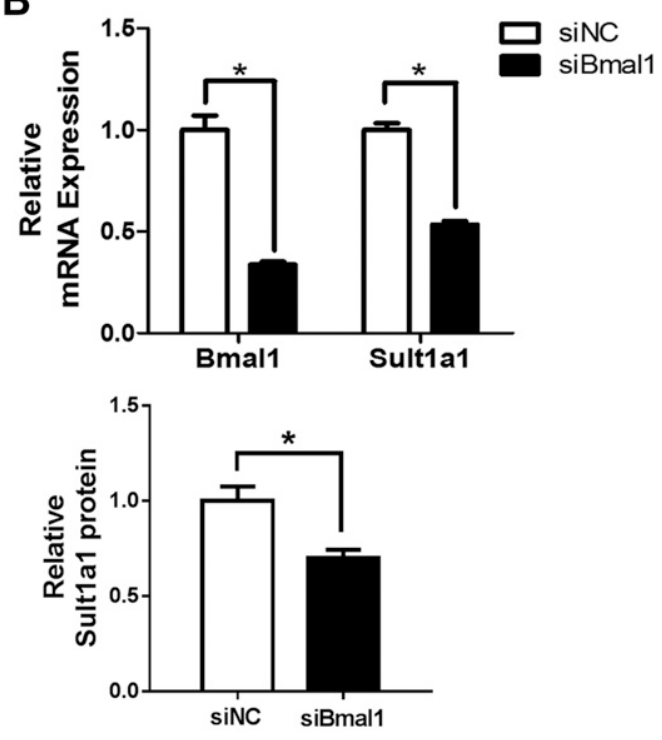

Fig. 3. Effects of Bmall overexpression (A) and knockdown (B) on Sult1a1 mRNA and protein in Hepa-1c1c7 cells. Hepa-1c1c7 cells were seeded onto six-well plates. The cells were transfected with Bmall expression plasmid or siBmal1 for 24 or 48 hours. The mRNA and protein levels were quantified by RT-qPCR and enzyme-linked immunosorbent assay, respectively. ${ }^{*} P<0.05$ ( $t$ test). 

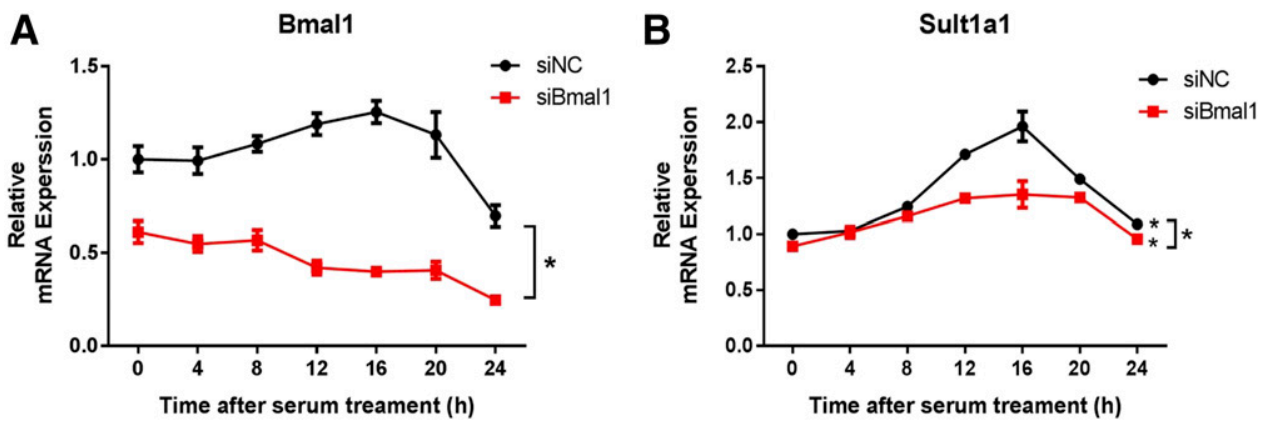
Fig. 4. Bmall knockdown blunts Sult1a1 rhythmicity in serum-shocked Hepa-1c1c7 cells. (A) mRNA level change of Bmal1 in Hepa-1c1c7 cells after siBmall transfection and serum shock. (B) mRNA level change of Sult1a1 in Hepa-1c1c7 cells after siBmal1 transfection and serum shock. $* P<0.05$ (one-way ANOVA or two-way ANOVA).

promote activity (Fig. 5, A and B). Fourth, Bmal1 protein bound directly to an E-box element (i.e., the -571- to -554-bp region) in Sult1al promoter based on a combination of promoter truncation, EMSA, and ChIP assays (Figs. 5 and 6). The evidence for regulation of rhythmic Sult1a1 by Bmal1 was also strong. Genetic deletion of Bmall in mice blunted the circadian rhythmicity of hepatic Sult1a1 (Fig. 2). Likewise, Bmal1 knockdown dampened the expression rhythmicity of Sult1a1 in serum-shocked Hepa-1c1c7 cells (Fig. 4).

It was a novel discovery that the core clock gene (Bmal1) can directly regulate Sult1a1 (a drug-processing gene) and is responsible for the enzyme's diurnal expression. This contrasted with an indirect role of core clock genes in circadian regulation of drug-processing genes such as Cyp2b10 and Mdrla in previous studies (Gachon et al., 2006; Murakami et al., 2008). In these studies, the clock output genes such as the PAR bZip transcription factors DBP, HLF, TEF (thyrotroph embryonic factor), and/or E4 promoter-binding protein 4 contribute extensively to circadian expressions and activities of Cyp2b10 and Mdr1a (Gachon et al., 2006; Murakami et al., 2008). For instance, circadian expression of Cyp2b10 was generated through the rhythmic Car (constitutive androstane receptor, a transcriptional activator of Cyp2b10) that was directly regulated by DBP, TEF, and HLF rather than the core clock genes (Gachon et al., 2006).

Hepatic Sult1a1 mRNA in mice peaked at the day-to-night transition time consistent with a previous study (Zhang et al., 2009). By contrast, the circadian protein profile showed a zenith at ZT18 (Fig. 1). A delay of 4-6 hours in the translation of mRNA to protein appeared to be within the normal range (Narumi et al., 2016). The finding that Sult1al with higher protein and activity levels during the night (the active phase for
A

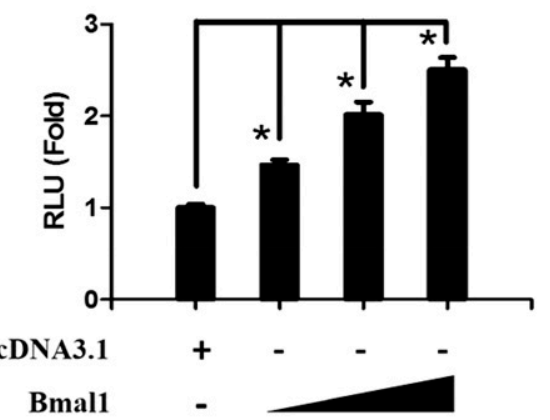

C

\section{Mouse Sult1a1 Promoter}

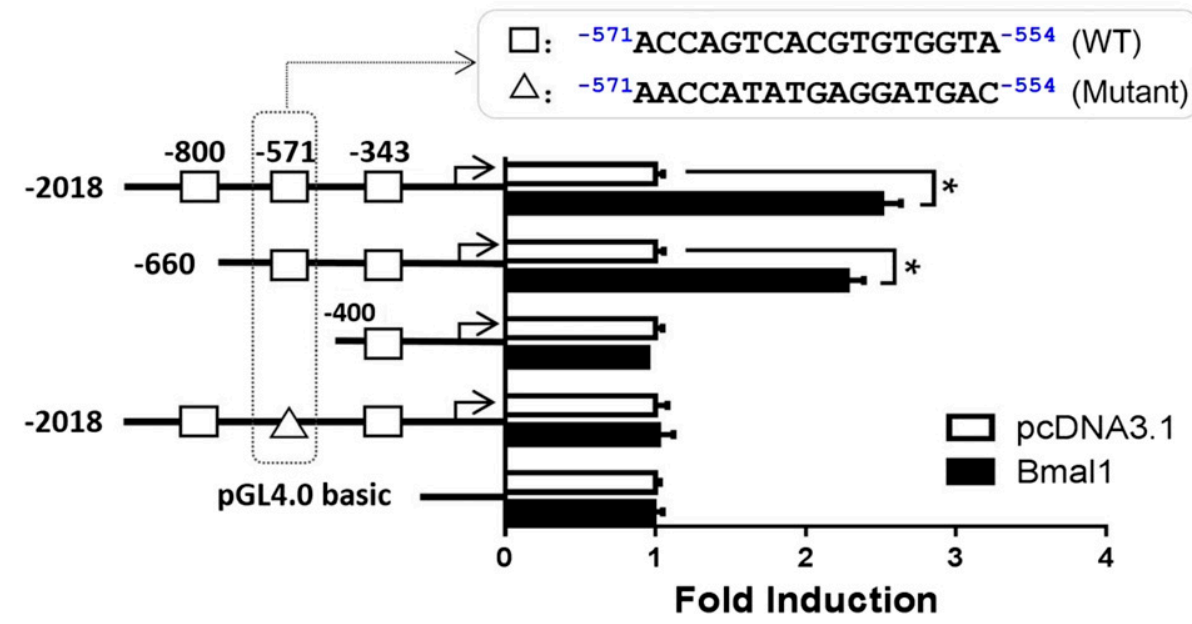

Fig. 5. Transcriptional activation of Sult1a1 by Bmal1 in luciferase reporter assays. (A) Bmal1 dose-dependently induces the Sult1al promoter activity. Hepa-1c1c7 cells were transfected with different amounts of Bmall expression plasmid. (B) Effects of Bmall knockdown on the Sult1a1 promoter activity. Hepa-1c1c7 cells were transfected with siBmall for 24 hours. (C) Effects of Bmall on the activities of different versions of Sult1a1 luciferase reporters. Hepa1c1c7 cells were cotransfected with different versions of Sultla1 luciferase reporters and Bmal1 expression plasmid for 24 hours. Data shown are the mean \pm S.D. $(n=5) . * P<0.05$ ( $t$ test). 
A

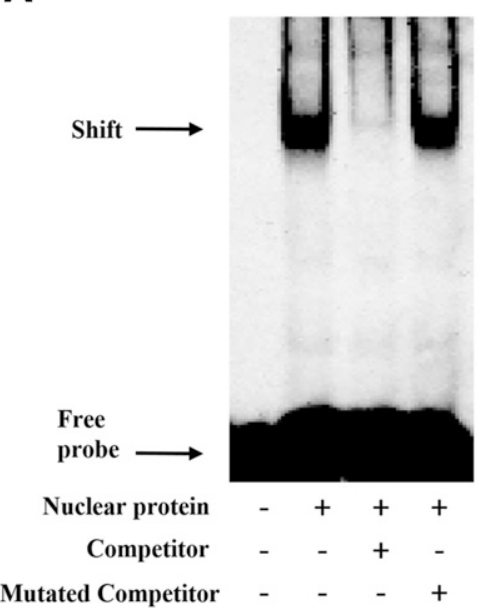

B

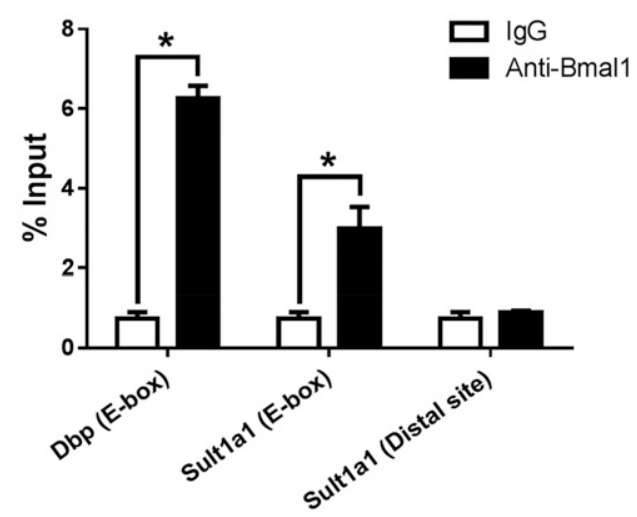

Fig. 6. Interactions of Bmall with Sultlal promoter. (A) Bmall binds to Sultlal promoter (-571- to -554-bp region, an E-box element). EMSAs were performed with labeled Sult1al promoter probe and/or competitors in the presence of nuclear extracts from Bmall-overexpressing Hepa-1c1c7 cells. (B) ChIP assay results, showing recruitment of Bmall protein to Sultlal promoter (571- to -554 -bp region, an E-box element). ${ }^{*} P<0.05$ ( $t$ test). mice) than the daytime (the rest phase for mice) was consistent with the notion that the body tends to develop a greater detoxification ability in the active phase with greater chances for xenobiotic challenges (Dallmann et al., 2016).

Identifying circadian genes among drug-processing genes and elucidating the circadian regulation mechanisms assume great importance in advancing chronopharmacokinetics (a discipline concerning time-dependent absorption, distribution, metabolism, and excretion) (Lemmer, 1999). Chronopharmacokinetics is strongly associated with chronotherapeutics that aim to find out the best timing (highest drug activity and least toxicity) for drug administration (Ballesta et al., 2017). Although a number of drug-processing genes have been shown to undergo circadian changes based solely on the mRNA determinations (Zhang et al., 2009), a full characterization of these genes at protein and activity levels is lacking. This is the first report revealing a phase II enzyme (Sult1a1) as a circadian gene at the protein and enzymatic activity (in vitro) level. However, due to the lack of an in vivo functional marker (or a compound exclusively metabolized by Sult1a1), we were unable to determine in vivo activity of Sult la1 and to characterize time dependency of Sult1a1 activity in vivo.

Since BMAL1 and CLOCK form a heterodimer to activate the transcription of many clock output genes including PER and $C R Y$, it is essential to test whether CLOCK also regulates Sult1a1. Surprisingly, Clock failed to induce Sult1a1 transcription in luciferase reporter assays and showed no effects on Bmall transactivation of Sult1a1 (data not shown), suggesting independent regulation of Sult1a1 by Bmal1. In fact, BMAL1 can independently regulate the expression of certain genes such as Dio2 (type II iodothyronine deiodinase) (Sawant et al., 2017). Although direct transcriptional activation of Sultla1 by Clock is unlikely, Clock may participate in regulation of Sult1al via forming a heterodimer with Bmall for promoter binding.

The serum shock method was employed herein to investigate the effect of Bmal1 on rhythmic expression of Sult1a1 at a cellular level because a serum shock (with a high concentration of serum) can induce circadian gene expression in the cells (Balsalobre et al., 1998). This is probably because in response to a high serum level, the serum response factor stimulates the expression of its target genes (including the core clock genes Per and Bmal1), thereby inducing circadian gene expression (Gerber et al., 2013). The method has been successfully used to study the molecular mechanisms for circadian regulation of several CYP enzymes including CYP2D6, CYP2E1, and CYP3A4 (Takiguchi et al., 2007; Matsunaga et al., 2008, 2012). Considering instantaneous effect of siRNA (for Bmal1 silencing), the serum shock experiments were conducted for 24 hours. Within this time period, both Bmal1 and Sult1a1 mRNA display obvious fluctuations (Fig. 4). The rhythmicity of Sult1a1 mRNA was strongly suppressed in Bmal1 knockdown cells, supporting a critical role of Bmal1 in circadian regulation of Sult1a1 (Fig. 4).

In summary, hepatic Sult1al was rhythmically expressed at both mRNA and protein levels. The clock protein Bmall transactivated Sult1a1, thereby controlling circadian expression of the enzyme.

\section{Authorship Contributions}

Participated in research design: Guo, Yu, Zhang, Wu.

Conducted experiments: Guo, Yu, Zhang.

Performed data analysis: Guo, Yu, Zhang, Wu.

Wrote the manuscript: Guo, Wu.

\section{References}

Akhtar RA, Reddy AB, Maywood ES, Clayton JD, King VM, Smith AG, Gant TW, Hastings MH, and Kyriacou CP (2002) Circadian cycling of the mouse liver transcriptome, as revealed by cDNA microarray, is driven by the suprachiasmatic nucleus. Curr Biol 12:540-550.

Allali-Hassani A, Pan PW, Dombrovski L, Najmanovich R, Tempel W, Dong A, Loppnau P, Martin F, Thornton J, Edwards AM, et al. (2007) Structural and chemical profiling of the human cytosolic sulfotransferases [published correction appears in PLoS Biol (2007) 5:e165]. PLoS Biol 5:e97.

Ballesta A, Innominato PF, Dallmann R, Rand DA, and Lévi FA (2017) Systems chronotherapeutics. Pharmacol Rev 69:161-199.

Balsalobre A, Damiola F, and Schibler U (1998) A serum shock induces circadian gene expression in mammalian tissue culture cells. Cell 93:929-937.

Blanchard RL, Freimuth RR, Buck J, Weinshilboum RM, and Coughtrie MW (2004) A proposed nomenclature system for the cytosolic sulfotransferase (SULT) superfamily. Pharmacogenetics 14:199-211

Buhr ED and Takahashi JS (2013) Molecular components of the Mammalian circadian clock. Handb Exp Pharmacol (217):3-27.

Chapman E, Best MD, Hanson SR, and Wong CH (2004) Sulfotransferases: structure, mechanism, biological activity, inhibition, and synthetic utility. Angew Chem Int Ed Engl 43:3526-3548.

Dallmann R, Okyar A, and Lévi F (2016) Dosing-time makes the poison: circadian regulation and pharmacotherapy. Trends Mol Med 22:430-445.

DeBruyne JP, Weaver DR, and Dallmann R (2014) The hepatic circadian clock modulates xenobiotic metabolism in mice. J Biol Rhythms 29:277-287.

Dong D, Ako R, and Wu B (2012) Crystal structures of human sulfotransferases: insights into the mechanisms of action and substrate selectivity. Expert Opin Drug Metab Toxicol 8:635-646.

Frame LT, Ozawa S, Nowell SA, Chou HC, DeLongchamp RR, Doerge DR, Lang NP, and Kadlubar FF (2000) A simple colorimetric assay for phenotyping the major human thermostable phenol sulfotransferase (SULT1A1) using platelet cytosols. Drug Metab Dispos 28: $1063-1068$.

Gachon F, Olela FF, Schaad O, Descombes P, and Schibler U (2006) The circadian PAR-domain basic leucine zipper transcription factors DBP, TEF, and HLF modulate basal and inducible xenobiotic detoxification. Cell Metab 4:25-36.

Gamage N, Barnett A, Hempel N, Duggleby RG, Windmill KF, Martin JL, and McManus ME (2006) Human sulfotransferases and their role in chemical metabolism. Toxicol Sci 90:5-22.

Gerber A, Esnault C, Aubert G, Treisman R, Pralong F, and Schibler U (2013) Blood-borne circadian signal stimulates daily oscillations in actin dynamics and SRF activity. Cell 152: 492-503.

Glatt H, Boeing H, Engelke CE, Ma L, Kuhlow A, Pabel U, Pomplun D, Teubner W, and Meinl W (2001) Human cytosolic sulphotransferases: genetics, characteristics, toxicological aspects. Mutat Res 482:27-40.

James MO and Ambadapadi S (2013) Interactions of cytosolic sulfotransferases with xenobiotics. Drug Metab Rev 45:401-414. 
Košir R, Španinger K, and Rozman D (2013) Circadian events in human diseases and in cytochrome P450-related drug metabolism and therapy. IUBMB Life 65:487-496.

Lemmer B (1999) Chronopharmacokinetics: implications for drug treatment. J Pharm Pharmacol 51:887-890

Lévi F, Tampellini M, Metzger G, Bizi E, Lemaigre G, and Hallek M (1994) Circadian changes in mitoxantrone toxicity in mice: relationship with plasma pharmacokinetics. Int $J$ Cancer 59: 543-547.

Lu D, Wang S, Xie Q, Guo L, and Wu B (2017) Transcriptional regulation of human UDPglucuronosyltransferase 2B10 by farnesoid X receptor in human hepatoma HepG2 cells. Mo Pharm 14:2899-2907.

Lu YF, Jin T, Xu Y, Zhang D, Wu Q, Zhang YK, and Liu J (2013) Sex differences in the circadian variation of cytochrome 450 genes and corresponding nuclear receptors in mouse liver. Chronobiol Int 30:1135-1143.

Matsunaga N, Ikeda M, Takiguchi T, Koyanagi S, and Ohdo S (2008) The molecular mechanism regulating 24-hour rhythm of CYP2E1 expression in the mouse liver. Hepatology 48:240-251.

Matsunaga N, Inoue M, Kusunose N, Kakimoto K, Hamamura K, Hanada Y, Toi A, Yoshiyama Y, Sato F, Fujimoto K, et al. (2012) Time-dependent interaction between differentiated embryo chondrocyte-2 and CCAAT/enhancer-binding protein $\alpha$ underlies the circadian expression of CYP2D6 in serum-shocked HepG2 cells. Mol Pharmacol 81:739-747.

Murakami Y, Higashi Y, Matsunaga N, Koyanagi S, and Ohdo S (2008) Circadian clock-controlled intestinal expression of the multidrug-resistance gene mdrla in mice. Gastroenterology 135 1636-1644.e3

Narumi R, Shimizu Y, Ukai-Tadenuma M, Ode KL, Kanda GN, Shinohara Y, Sato A, Matsumoto $\mathrm{K}$, and Ueda HR (2016) Mass spectrometry-based absolute quantification reveals rhythmic variation of mouse circadian clock proteins. Proc Natl Acad Sci USA 113:E3461-E3467.

Ono D, Honma K, and Honma S (2015) Circadian and ultradian rhythms of clock gene expression in the suprachiasmatic nucleus of freely moving mice. Sci Rep $\mathbf{5}: 12310$

Otake Y, Hsieh F, and Walle T (2002) Glucuronidation versus oxidation of the flavonoid galangin by human liver microsomes and hepatocytes. Drug Metab Dispos 30:576-581.

Pappa KI, Gazouli M, Anastasiou E, Iliodromiti Z, Antsaklis A, and Anagnou NP (2013) The major circadian pacemaker ARNT-like protein-1 (BMAL1) is associated with susceptibility to gestational diabetes mellitus. Diabetes Res Clin Pract 99:151-157.

Partch CL, Green CB, and Takahashi JS (2014) Molecular architecture of the mammalian circadian clock. Trends Cell Biol 24:90-99.

Reppert SM and Weaver DR (2002) Coordination of circadian timing in mammals. Nature 418 935-941.

Richards J, Diaz AN, and Gumz ML (2014) Clock genes in hypertension: novel insights from rodent models. Blood Press Monit 19:249-254.

Richardson SJ, Bai A, Kulkarni AA, and Moghaddam MF (2016) Efficiency in drug discovery: liver S9 fraction assay as a screen for metabolic stability. Drug Metab Lett 10:83-90.
Riches Z, Stanley EL, Bloomer JC, and Coughtrie MW (2009) Quantitative evaluation of the expression and activity of five major sulfotransferases (SULTs) in human tissues: the SULT "pie". Drug Metab Dispos 37:2255-2261.

Sawant OB, Horton AM, Zucaro OF, Chan R, Bonilha VL, Samuels IS, and Rao S (2017) The circadian clock gene Bmall controls thyroid hormone-mediated spectral identity and cone photoreceptor function. Cell Reports 21:692-706.

Shimba S, Ogawa T, Hitosugi S, Ichihashi Y, Nakadaira Y, Kobayashi M, Tezuka M, Kosuge Y, Ishige K, Ito Y, et al. (2011) Deficient of a clock gene, brain and muscle Arnt-like protein-1 (BMAL1), induces dyslipidemia and ectopic fat formation. PLoS One 6:e25231.

Solt LA, Kojetin DJ, and Burris TP (2011) The REV-ERBs and RORs: molecular links between circadian rhythms and lipid homeostasis. Future Med Chem 3:623-638.

Stratmann M, Suter DM, Molina N, Naef F, and Schibler U (2012) Circadian Dbp transcription relies on highly dynamic BMAL1-CLOCK interaction with $\mathrm{E}$ boxes and requires the proteasome. Mol Cell 48:277-287.

Sukumaran S, Almon RR, DuBois DC, and Jusko WJ (2010) Circadian rhythms in gene expression: relationship to physiology, disease, drug disposition and drug action. Adv Drug Deliv Rev 62:904-917.

Sun H, Ma Z, Lu D, and Wu B (2015) Regio- and isoform-specific glucuronidation of psoralidin: evaluation of 3-o-glucuronidation as a functional marker for UGT1A9. J Pharm Sci 104:2369-2377. Takahashi JS, Hong HK, Ko CH, and McDearmon EL (2008) The genetics of mammalian circadian order and disorder: implications for physiology and disease. Nat Rev Genet 9:764-775

Takiguchi T, Tomita M, Matsunaga N, Nakagawa H, Koyanagi S, and Ohdo S (2007) Molecular basis for rhythmic expression of CYP3A4 in serum-shocked HepG2 cells. Pharmacogenet Genomics 17:1047-1056.

Teubner W, Meinl W, Florian S, Kretzschmar M, and Glatt H (2007) Identification and localization of soluble sulfotransferases in the human gastrointestinal tract. Biochem J 404:207-215.

Wang S, Yuan X, Lu D, Guo L, and Wu B (2017) Farnesoid X receptor regulates SULT1E1 expression through inhibition of PGC1 $\alpha$ binding to HNF4 $\alpha$. Biochem Pharmacol 145:202-209.

Zhang T, Zhao M, Lu D, Wang S, Yu F, Guo L, Wen S, and Wu B (2018) REV-ERB $\alpha$ regulates CYP7A1 through repression of liver receptor homolog-1. Drug Metab Dispos 46:248-258.

Zhang YK, Yeager RL, and Klaassen CD (2009) Circadian expression profiles of drug-processing genes and transcription factors in mouse liver. Drug Metab Dispos 37:106-115.

Zhao M, Zhang T, Yu F, Guo L, and Wu B (2018) E4bp4 regulates carboxylesterase 2 enzymes through repression of the nuclear receptor Rev-erb $\alpha$ in mice. Biochem Pharmacol 152:293-301.

Address correspondence to: Dr. Baojian Wu, College of Pharmacy, Jinan University, 601 Huangpu Avenue West, Guangzhou 510632, China. E-mail: bj.wu@ hotmail.com 